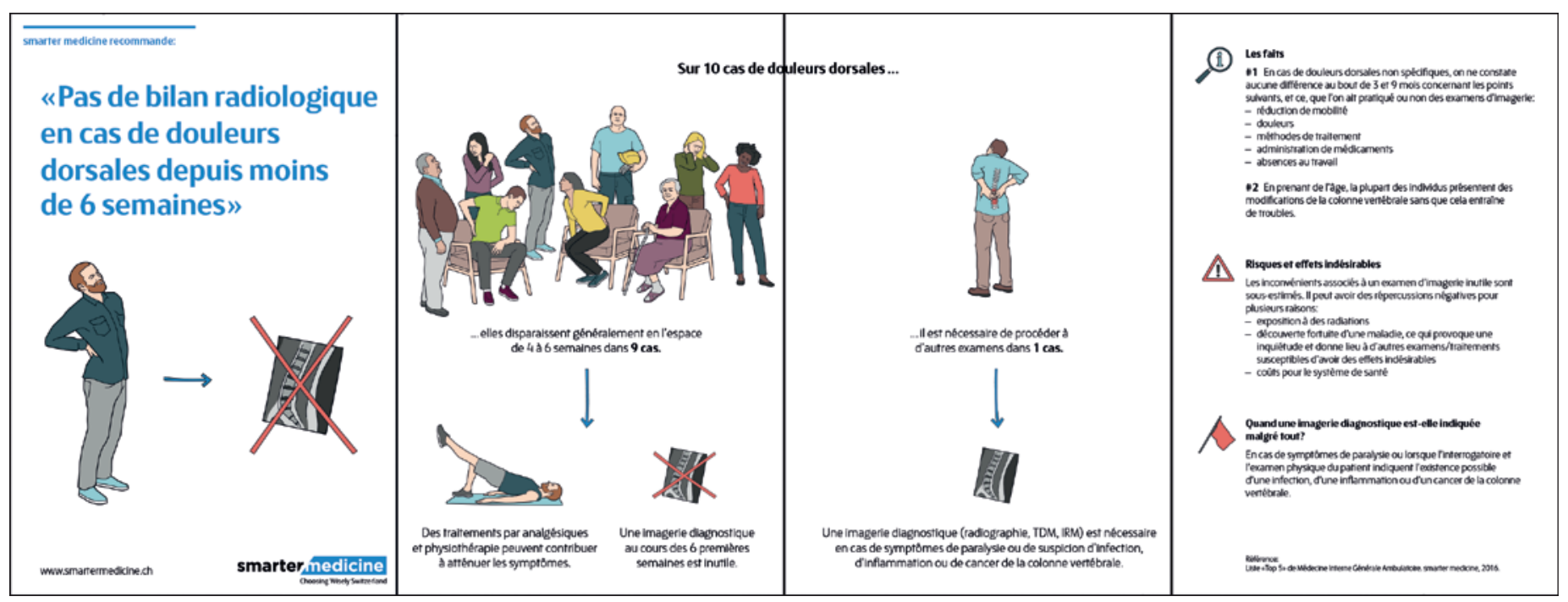

Les infographies peuvent être remises aux patients sous forme de dépliants et sont téléchargeables gratuitement en PDF sur: www.smartermedicine.ch/fr/pour-specialistes/infografiken-fuer-behandelnde.html (grafique: ๑ smarter medicine - Choosing Wisely Switzerland).

\title{
Surmédicalisation: infographies pour le dialogue avec les patients
}

\section{Lars Clarfelda, Hermann Amstad}

a Dr méd. Lars Clarfeld, Secrétaire général de la SSMIG et smarter medicine; ${ }^{\text {b }}$ Dr méd., amstad-kor, Bâle

Aujourd'hui, la médecine offre de nombreuses options. Mais tout ce qui serait possible n'a pas forcément de sens dans tous les cas. Selon la devise «less is more», l'association «smarter medicine» lutte contre la surmédicalisation et les soins inappropriés en médecine. En collaboration avec le «Swiss Medical Board», l'association a réalisé des infographies pour sensibiliser les patients à la qualité des traitements et fournir au corps médical un outil pour la pratique quotidienne.

Le système de santé suisse est surmédicalisé: Selon une étude commandée par l'Académie Suisse des Sciences Médicales en 2012, entre 20 et 30\% des interventions médicales sont superflues [1]. Cette pratique inappropriée et cette surmédicalisation ont un impact négatif sur la qualité des soins et leurs coûts [2]. Ce fait relève également, mais pas seulement, de la responsabilité du corps médical: certains patients attendent ou même exigent une certaine clarification ou thérapie, même si elle n'est pas médicalement appropriée. Informer les patients sur des traitements potentiellement dangereux ou dénués de sens peut être exigeant et prendre beaucoup de temps, tant pour le médecin traitant que pour le patient ou la patiente. Il existe un risque que les patients aient le sentiment de ne pas être pris au sérieux et changent de médecin. 


\section{Infographie contre les surmédicalisations et des soins inappropriés}

Le Swiss Medical Board (SMB) et smarter medicine sont deux initiatives visant à réduire les développements indésirables concernant la surmédicalisation et les soins inappropriés au sein du système de santé suisse.

\section{Le patient peut bénéficier d'une explication simple des faits.}

Avec leurs rapports, leurs campagnes et leurs recommandations, ils contribuent à trouver le meilleur traitement pour chaque patient, conformément à la devise «la médecine optimale, et non pas maximale».

Compte tenu des problèmes décrits ci-dessus et selon les rapports et recommandations, il a été décidé d'établir des outils destinés aux praticiens, appelés «infographies", pour certaines recommandations et rapports. Ces infographies soutiennent la profession médicale dans sa communication avec les patients; elles sont avant tout destinées à des recommandations dont la pertinence pour les patients est considérée comme élevée, c'est-à-dire celles pour lesquelles les patients ont une certaine attente. Selon l'International Health Policy Survey 2019 du Fonds du Commonwealth, les attentes des patients et le manque de temps pour la prise de décision conjointe sont les principaux obstacles pour éviter les traitements inappropriés. C'est précisément dans ce domaine que l'infographie est censée apporter un soutien en tant que solution. Le patient peut bénéficier d'une explication simple des faits et bénéficier d'informations complémentaires «objectives» et indépendantes.

Des outils similaires sont déjà connus de l'étranger. Par exemple, le Centre Harding pour la compétence en ma-

\section{L'essentiel en bref}

- La pratique inappropriée et la surmédicalisation ont un impact négatif sur la qualité des soins et leurs coûts

- Non seulement les médecins mais aussi les patients jouent un rôle important dans cette situation.

- C'est pourquoi les associations "Smarter Medicine " et " Swiss Medical Board " ont commandé le développement d'aides pour les personnes qui dispensent des traitements, appelées «infographies», afin de communiquer au patient des questions complexes de manière simple.

- II en résulte une infographie pour chacun des domaines de la prévention (test PSA), du diagnostic (imagerie pour les douleurs dorsales) et de la thérapie (antibiotiques pour les infections des voies respiratoires supérieures).

- Les médecins sont invités à partager leurs expériences et toute suggestion d'amélioration avec le Secrétariat de smarter medicine via smartermedicine[at]sgaim.ch. tière de risques à Berlin, dirigé par le professeur Gerd Gigerenzer, a développé des "fiches d'information". Ces fiches d'information sont conçues pour permettre aux patients de comprendre les avantages et les inconvénients de certaines mesures médicales afin qu'ils puissent décider eux-mêmes de les subir ou non [3]. Ces fiches d'information sont évaluées scientifiquement en ce qui concerne l'efficacité de la communication des connaissances pertinentes pour les patients et les décisions de santé [4].

\section{Coopération interprofessionnelle au service des patients}

En automne 2018, les associations Swiss Medical Board et smarter medicine ont chargé un groupe de travail de développer de tels outils. Le groupe de travail était composé d'experts dans les domaines de la médecine hospitalière, de la médecine ambulatoire, des professions de santé non médicales, de l'épidémiologie, de l'éthique, de la protection des consommateurs et, enfin et surtout, d'experts en communication et en graphisme. Cette approche interprofessionnelle vise à améliorer la qualité de l'infographie.

\section{Les trois infographies sont structurées de} manière similaire en ce sens que chacune se compose d'une feuille de papier A4 pliée.

En coopération avec une agence de communication, le groupe de travail a développé des infographies exemplaires dans les domaines de la prévention (test PSA), du diagnostic (imagerie pour les douleurs dorsales) et de la thérapie (antibiotiques pour les infections des voies respiratoires supérieures). Le groupe de travail a basé son contenu sur la littérature qui sous-tend les recommandations de smarter medicine et - dans le cas du test PSA - sur le rapport du SMB et la littérature actuelle.

Les trois infographies sont structurées de manière similaire en ce sens que chacune se compose d'une feuille de papier A4 pliée et contienne une visualisation du message central dans la partie intérieure et au verso, dans les trois sections «Faits», «Risques et effets secondaires» et «Exceptions». Les informations essentielles sont également présentées sous forme écrite concise.

Les trois prototypes d'infographies qui en résultent on été vérifiés au cours d'entretiens avec des profanes, puis adaptés et sont maintenant disponibles en allemand, en français et en italien. La figure 1 montre un exemple de l'avant et de l'arrière des graphiques d'information permettant de renoncer à l'imagerie diagnostique dans les cas de douleurs dorsales de courte durée. 
L'accès visuel aux infographies permet de faciliter la communication avec les patients dans différents contextes. Elles transmettent les faits complexes de manière simple et non verbale et expliquent pourquoi il est préférable pour lui de s'abstenir de tout traitement médical et quels sont les risques associés aux différents traitements. De cette manière, elles simplifient la communication entre le professionnel et le patient, servent à «habiliter» le patient et améliorent ainsi la qualité du traitement.

\section{Les infographies sont désormais disponibles gratuitement sur les sites web de smarter medicine et du Swiss Medical Board.}

C'est du moins ce qu'attendent les initiateurs; une étude serait nécessaire pour prouver directement l'effet, et une telle étude n'est pas encore prévue pour le moment en raison des coûts élevés qu'elle implique. Les preuves indirectes peuvent au mieux être trouvées dans un projet de recherche du Prof. Matthias Schwenkglenks et al. dans le cadre du PNR 74 Système de santé; sous le titre "Influence des directives et des recommandations sur les traitements médicaux", le projet examinera s'il existe un lien entre le choix du traitement et les directives et recommandations médicales disponibles [5]. Entre-temps, les organes compétents du Swiss Medical Board et de smarter medicine ont examiné les trois «prototypes» et les ont approuvés pour publication. Les infographies sont désormais disponibles gratuitement et à tout moment sur les sites web de smarter medicine et du Swiss Medical Board.

\section{Le feedback de la profession médicale est souhaité}

Dans une prochaine phase, les infographies seront soumises à un test pratique. Les médecins sont invités à partager leurs expériences et leurs éventuelles suggestions d'amélioration avec le Secrétariat de smarter medicine (smartermedicine[at]sgaim.ch). Si les trois prototypes sont acceptés et font leurs preuves dans la pratique quotidienne, le Swiss Medical Board et smarter medicine commanderont d'autres infographies; les sociétés de discipline peuvent également faire élaborer les infographies pour des interventions, en particulier pour les recommandations des listes Top 5. Ce serait alors également le moment opportun pour faire effectuer une étude d'évaluation.

\section{Télécharger}

Les infographies sont disponibles gratuitement sous forme de fichiers PDF à télécharger sur: www.smartermedicine.ch/fr/ pour-specialistes/infografiken-fuer-behandelnde.html

\section{Références}

1 Trageser J, Vettori A, Iten R, Criveli L. Effizienz, Nutzung und Finanzierung des Gesundheitswesens. Akademien der Wissenschaften Schweiz, Berne 2012.

2 Gerber M, Kraft E, Bosshard C. Overuse - unnötige Behandlungen als Qualitätsproblem. Bull Med Suisses. 2016;97:236-43.

3 https://www.hardingcenter.de/de/projekte-und-kooperationen/ faktenboxen

4 Loizeau AJ, et al. Fact Box decision support tools reduce decisional conflict about antibiotics for pneumonia and artificial hydration in advanced dementia: A randomized controlled trail. Age and Ageing. 2019;48:67-74

5 http://www.nfp74.ch/fr/projets/soins-multisectoriels/projet-schwenkglenks

\section{L'association smarter medicine}

L'association de parrainage smarter medicine-Choosing Wisely Switzerland a été fondée en juin 2017 et fait suite à l'initiative américaine "Choosing Wisely", qui a connu un grand succès. Son but n'est pas seulement de susciter des "décisions sages», mais aussi de promouvoir un débat ouvert entre le corps médical, les patients et le public. Outre la Société Suisse de Médecine Interne Générale (SGAIM) et I'Académie Suisse des Sciences Médicales (ASSM), I'association est soutenue par l'Association suisse des organisations professionnelles de santé (svbg), I'Organisation faîtière des associations suisses de patients (DVSP) et les organisations de consommateurs Stiftung für Konsumentenschutz (SKS), Fédération Romande des Consommateurs (FRC) et Associazione Consumatrici e Consumatori della Svizzera Italiana (acsi). A ce jour, quatorze listes Top 5 sur les traitements inappropriés ont été publiées par des associations professionnelles de médecins et d'infirmiers. D'autres associations professionnelles sont invitées à rejoindre le mouvement.
Dr méd. Lars Clarfeld

Directeur

smarter medicine Secretaire général SSMIC Monbijoustrasse 43 Case postale CH-3001 Berne lars.clarfeld[at]sgaim.ch

\section{Swiss Medical Board}

Le Swiss Medical Board est un centre de compétence suisse de premier plan pour l'évaluation des technologies de la santé. II analyse et évalue les procédures préventives et diagnostiques ainsi que les interventions thérapeutiques du point de vue de la médecine, de l'économie, de l'éthique et du droit.

Le Swiss Medical Board est soutenu par une association dont les institutions et sociétés suivantes sont membres: Conférence des directeurs cantonaux de la santé (GDK), Académie
Suisse des Sciences Médicales (ASSM), Gouvernement de la Principauté de Liechtenstein, Association des entreprises suisses de recherche pharmaceutique (interpharma), Associations des assureurs-maladie (santésuisse, curafutura), Fondation suisse SPO Protection des brevets et Association faîtière des centres suisses pour patients (DVSP), Association faîtière des sociétés spécialisées en chirurgie et en chirurgie invasive (fmCh). 